\title{
Tunneling magnetoresistance in Fe/MgO granular multilayers
}

\author{
A. García-García, ${ }^{1,2, a)}$ A. Vovk ${ }^{2,3,4}$ J. A. Pardo, ${ }^{3,5}$ P. Štrichovanec, ${ }^{3}$ P. A. Algarabel, ${ }^{1,2}$ \\ C. Magén, ${ }^{6}$ J. M. De Teresa, ${ }^{1,2}$ L. Morellón, ${ }^{1,2,3}$ and M. R. Ibarra ${ }^{1,2,3}$ \\ ${ }_{1}^{1}$ Instituto de Ciencia de Materiales de Aragón, Universidad de Zaragoza-CSIC, 50009 Zaragoza, Spain \\ ${ }^{2}$ Departamento de Física de la Materia Condensada, Universidad de Zaragoza, 50009 Zaragoza, \\ Spain \\ ${ }^{3}$ Instituto de Nanociencia de Aragón, Universidad de Zaragoza, 50018 Zaragoza, Spain \\ ${ }^{4}$ Institute of Magnetism, NAS of Ukraine, 36-b Vernnadsky blvd., 03142 Kyiv, Ukraine \\ ${ }^{5}$ Departamento de Ciencia y Tecnología de Materiales y Fluidos, Universidad de Zaragoza, \\ 50018 Zaragoza, Spain \\ ${ }^{6}$ Instituto de Nanociencia de Aragón-ARAID, Universidad de Zaragoza, 50018 Zaragoza, Spain
}

(Received 22 December 2009; accepted 2 January 2010; published online 1 February 2010)

\begin{abstract}
We have investigated the transport and magnetotransport properties in $\mathrm{Fe} / \mathrm{MgO}$ multilayers around the Fe percolation threshold as a function of the temperature and the nominal thickness of iron layer (t). Electrical resistivity measurements allowed us to disclose the charge transport mechanisms involved, which are closely related to the degree of discontinuity in the Fe layers. The samples with Fe thickness below percolation threshold $(\mathrm{t} \sim 0.8 \mathrm{~nm})$ exhibit isotropic magnetoresistance $(\mathrm{MR})$, which can be understood considering spin-polarized electron tunneling between nanometer-sized, superparamagnetic Fe grains. The MR ratio increases with decreasing temperature from $\sim 3 \%$ at room temperature to $\sim 10 \%$ at $30 \mathrm{~K}$. The temperature dependence of $\mathrm{MR}$ can be explained satisfactorily in terms of a modified Mitani's model. (C) 2010 American Institute of Physics.

[doi:10.1063/1.3298504]
\end{abstract}

A new generation of devices combining the advantages of semiconductor electronics with spin-dependent transport is emerging as a consequence of the strong potential for magnetoelectronic applications, such as storage media, sensors, and potentiometers. ${ }^{1}$ The interest in the development of these spintronic devices inspired the search for new materials in the nanometric length scale, where the magnetic and transport properties differ substantially from those of the bulk. Among them we can mention granular cermets-in which nanometer-sized magnetic metallic particles are embedded in an insulating matrix. ${ }^{2-5}$

Discontinuous metal-insulator multilayers (DMIMs) are a special kind of granular cermets where the metallic particles are located not randomly but in layers, and this arrangement causes the enhancement of the low-field dependence and relatively high magnetoresistance (MR) ratios at room temperature. ${ }^{6}$ DMIMs are easier to prepare than planar, epitaxial magnetic tunnel junctions (MTJs) showing huge MR values, ${ }^{7,8}$ but strongly reduced by structural defects at interfaces. ${ }^{9,10}$ The physical principle that underlies the MR in both cases is a spin-dependent electric charge transfer between two magnetized electrodes or grains via tunneling effect. $^{11,12}$ This effect is called tunneling MR (TMR), and the tunneling probability depends on the relative orientation of magnetization of the electrodes or grains. ${ }^{13}$ Experimentally TMR ratios up to $180 \%$ in fully epitaxial $\mathrm{Fe}(001) /$ $\mathrm{MgO}(001) / \mathrm{Fe}(001)$ junction were found at room temperature, ${ }^{7}$ and first-principles calculations predict even higher values. ${ }^{14}$ Despite extensive research on planar $\mathrm{Fe} /$ $\mathrm{MgO} / \mathrm{Fe}$ MTJs, little attention has been paid to $\mathrm{Fe} / \mathrm{MgO}$

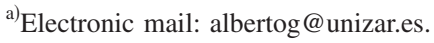

granular cermets. ${ }^{15,16}$ In this paper we present our results on the magnetotransport properties of $\mathrm{Fe} / \mathrm{MgO}$ DMIMs prepared by pulsed laser deposition (PLD).

Polycrystalline $[\mathrm{Fe}(\mathrm{t}) / \mathrm{MgO}(3 \mathrm{~nm})]_{\mathrm{N}}$ multilayers with $0.4 \mathrm{~nm} \leq \mathrm{t} \leq 1.5 \mathrm{~nm}$ were deposited by PLD on glass substrates. The preparation procedure was reported in detail elsewhere. ${ }^{16}$ Electrical resistance and MR measurements were carried out using the four-point method in the temperature range 30-295 $\mathrm{K}$ and in-plane applied magnetic field $(\mathrm{H})$ up to $18 \mathrm{kOe}$. Two different configurations of $\mathrm{H}$ with respect to current-in-plane (I) were studied: (1) H parallel to I (Lgeometry) and (2) $\mathrm{H}$ perpendicular to I (T-geometry). The MR ratio is defined as $\mathrm{MR}=[\rho(\mathrm{H})-\rho(0)] / \rho(0)$, where $\rho(\mathrm{H})$ and $\rho(0)$ are the resistivities of the film in magnetic field and at the demagnetized state, respectively.

It was found ${ }^{16}$ that the morphology of Fe layers evolves from a continuous film to a set of grains through percolation structures with decreasing t. Magnetization measurements show that multilayers with $\mathrm{t} \geq 0.81 \mathrm{~nm}$ are ferromagnetic, while those with $\mathrm{t} \leq 0.61 \mathrm{~nm}$ are superparamagnetic. The fit of the magnetization by using weighted Langevin functions and an approach of log-normal distribution of spherical particles reveals that average size for the superparamagnetic grains increases with $\mathrm{t}$ from $\approx 1.6 \mathrm{~nm}(\mathrm{t}=0.4 \mathrm{~nm})$ to $\approx 2.8 \mathrm{~nm}(\mathrm{t}=0.61 \mathrm{~nm})$. These data are in good agreement with direct transmission electron microscopy (TEM) observations. ${ }^{16}$ Moreover a decrease in the saturation magnetization $\left(M_{\mathrm{s}}\right)$ associated with formation of an ultrathin $\mathrm{Fe}$ oxide shell was found. ${ }^{16}$

The dependence of the electrical resistivity $(\rho)$ versus $t$ at room temperature (not shown) presents a sharp increase from $\rho=1540 \Omega \mathrm{cm}$ for $\mathrm{t}=0.81$, to $\rho=2.42 \times 10^{6} \Omega \mathrm{cm}$ for $\mathrm{t}=0.61 \mathrm{~nm}$, suggesting a transition from a continuous to a 


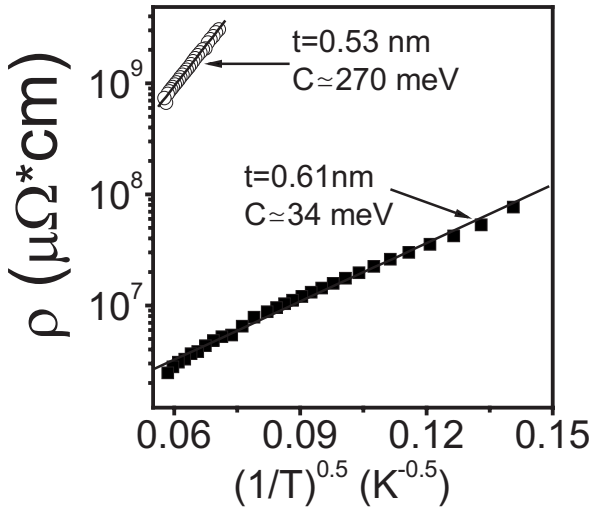

FIG. 1. Temperature dependence of the zero-field resistivity $\rho$ for $[\mathrm{Fe}(\mathrm{t}) / \mathrm{MgO}(3 \mathrm{~nm})]_{\mathrm{N}}$ multilayers. The Fe nominal thickness $(\mathrm{t})$ is indicated in the figure. Solid lines are the best fits to experimental data using the expression $\rho=\rho_{0} \exp \left[2\left(C / k_{B} T\right)^{1 / 2}\right]$.

discontinuous structure of the Fe layer and consequently a change in the transport mechanism, from metallic to tunneling conductance, respectively. This was directly confirmed by the temperature dependence of the resistivity, as shown below. The resistivity of the films with continuous Fe layers is higher than that of the bulk $\mathrm{Fe}(222 \mu \Omega \mathrm{cm}$ for $\mathrm{t}$ $=1.5 \mathrm{~nm}$ and $9.7 \mu \Omega \mathrm{cm}$ for bulk Fe). This effect has been attributed to the thickness confinement effects associated with the decrease in the electron mean free path and surface scattering in thin films. ${ }^{17,18}$

The temperature dependence of $\rho$ for two samples with $\mathrm{t} \leq 0.61 \mathrm{~nm}$ is shown in Fig. 1. The exponential law $\rho$ $=\rho_{0} \exp \left[2\left(C / k_{B} T\right)^{1 / 2}\right]$ characteristic of thermally assisted tunneling conductance in granular cermets ${ }^{3}$ is held. Here $\rho_{0}$ is the resistivity at infinite temperature, $\mathrm{C}$ is an effective activation energy defined as $C=2 \chi s E_{\mathrm{c}}$, where $\chi$ $=\left(2 m^{*} \phi \hbar^{2}\right)^{1 / 2}, \phi=U-E_{\mathrm{F}}$ is the effective barrier height, $m^{*}$ is the effective mass of electrons, $s$ is the mean separation of $\mathrm{Fe}$ particles, $E_{\mathrm{c}}$ is the energy necessary to generate a pair of neighboring charged grains during tunneling process, and $k_{B}$ is Boltzmann's constant. The value of $C$ increases with decreasing $\mathrm{t}$ as $C \approx 34 \mathrm{meV}$ for $\mathrm{t}=0.61 \mathrm{~nm}$ and $C$ $\approx 270 \mathrm{meV}$ for $\mathrm{t}=0.53 \mathrm{~nm}$. This is due to the decrease of the Fe particle size and increase in barrier thickness. As a first approximation it is possible to estimate the average interparticle separation $(s)$-to-diameter $(d)$ ratio using relation ${ }^{3}$ $C \approx 2 \chi e^{2}(s / d)^{2} / \varepsilon[1 / 2+(s / d)]$, where $e$ is the electron charge and $\varepsilon=9.7$ the dielectric constant of $\mathrm{MgO}$. The values of $d$ were obtained from fitting of magnetization loops ${ }^{16}$ as 2.2 and $2.8 \mathrm{~nm}$ for $\mathrm{t}=0.53$ and $\mathrm{t}=0.61 \mathrm{~nm}$, respectively. If we take $\chi=1 \AA^{-1}, 3,6$ the estimation using these parameters gives the average values $s \approx 2.1$ and $s \approx 1.1 \mathrm{~nm}$, respectively. These values are smaller than the nominal MgO thickness between adjacent $\mathrm{Fe}$ layers $(3 \mathrm{~nm})$. Thus, we propose that tunneling takes place along the chains of $\mathrm{Fe}$ granules within the same layer. It should be noted that although this approach is very rough as it does not take into account the particle size distribution, high order tunneling processes and the fact that dielectric constant in thin film could be different from the bulk, it gives a reasonable estimation of the $s$ values that correlates with direct TEM observations. ${ }^{16}$

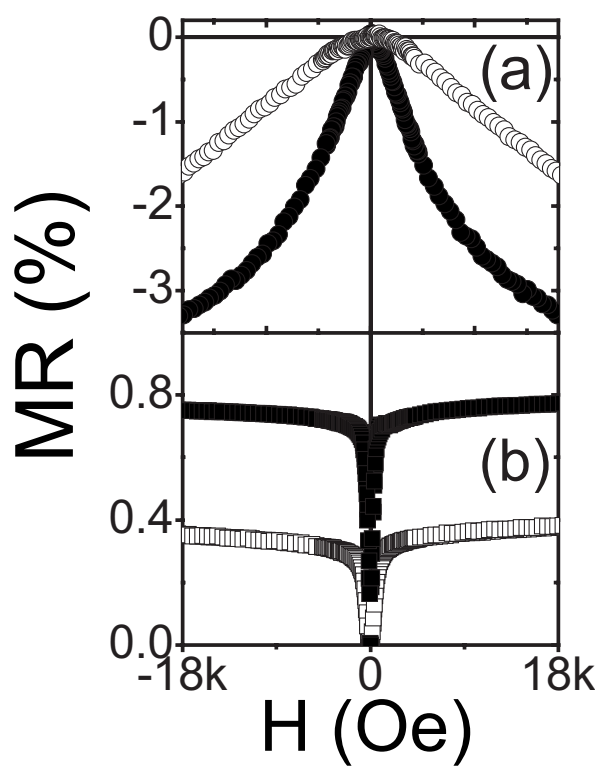

FIG. 2. Room-temperature dependencies of MR measured in L-geometry on applied magnetic field $(\mathrm{H})$ in $[\mathrm{Fe}(\mathrm{t}) / \mathrm{MgO}(3 \mathrm{~nm})]_{\mathrm{N}}$ multilayers, (a) discontinuous samples with $\mathrm{t}=0.61 \mathrm{~nm}$ (full circles) and $\mathrm{t}=0.53 \mathrm{~nm}$ (open circles), (b) Samples with continuous Fe layers with $t=1.25 \mathrm{~nm}$ (full squares) and $\mathrm{t}=0.81 \mathrm{~nm}$ (open squares).

The room temperature MR for several thicknesses is presented in Fig. 2. For $t \leq 0.61 \mathrm{~nm}$, the resistivity of the film decreases with increasing field and cannot be saturated at 18 kOe [Fig. 2(a)]. This is the typical behavior for TMR effect in granular cermets. ${ }^{5}$ The absolute value of TMR at $18 \mathrm{kOe}$ increases with $\mathrm{t}$ from $1.5 \% \quad(\mathrm{t}=0.53 \mathrm{~nm})$ to $3.3 \% \quad(\mathrm{t}$ $=0.61 \mathrm{~nm}$ ), which is associated with the increase of particle size and decrease of interparticle distance. ${ }^{19,20}$ We notice that the TMR ratios at room temperature are markedly reduced in comparison with the reported values for epitaxial $\mathrm{Fe}(001) /$ $\mathrm{MgO}(001) / \mathrm{Fe}(001)$ MTJs (Ref. 7) because the polycrystalline nature of our samples (seen in TEM images ${ }^{16}$ ) prevents the huge TMR values associated with band symmetry filtering occurring in epitaxial junctions. Furthermore, it is well known ${ }^{21}$ that in continuous ferromagnetic films the MR in L-geometry is positive and saturates at low fields. This type of behavior was found in the samples with $\mathrm{t} \geq 0.81 \mathrm{~nm}$ [see Fig. 2(b)].

The temperature dependence of TMR in granular cermets can be explained using the model of Mitani et al. ${ }^{22}$ This model takes into account higher-order processes of spindependent tunneling between large granules through intervening small ones with strong Coulomb blockade. In this case, for small values of $P^{2}(P$ is spin polarization of tunneling electrons) the temperature dependence of TMR could be approximated by expression TMR $=-P^{2} m^{2}(1+\sqrt{B / T})$, where $m=M / M_{\mathrm{S}}$ is the magnetization of the system normalized to the saturation magnetization $M_{\mathrm{s}}$, and $B$ is defined as $B$ $=E_{\mathrm{c}} /(8 s \chi)$. Zhu and Wang ${ }^{23}$ observed that this model cannot explain the TMR data obtained for their $\mathrm{Fe}-\mathrm{Al}_{2} \mathrm{O}_{3}$ granular cermets in 5-295 $\mathrm{K}$ temperature range and modified the expression of Mitani et al. ${ }^{22}$ adding a factor $\exp \left(-k_{B} T / E_{\mathrm{mr}}\right)$, where $E_{\mathrm{mr}}$ is an energy value associated with spin-flip scattering processes which can change the spin of the electrons involved in tunneling event between Fe grains. The modified 

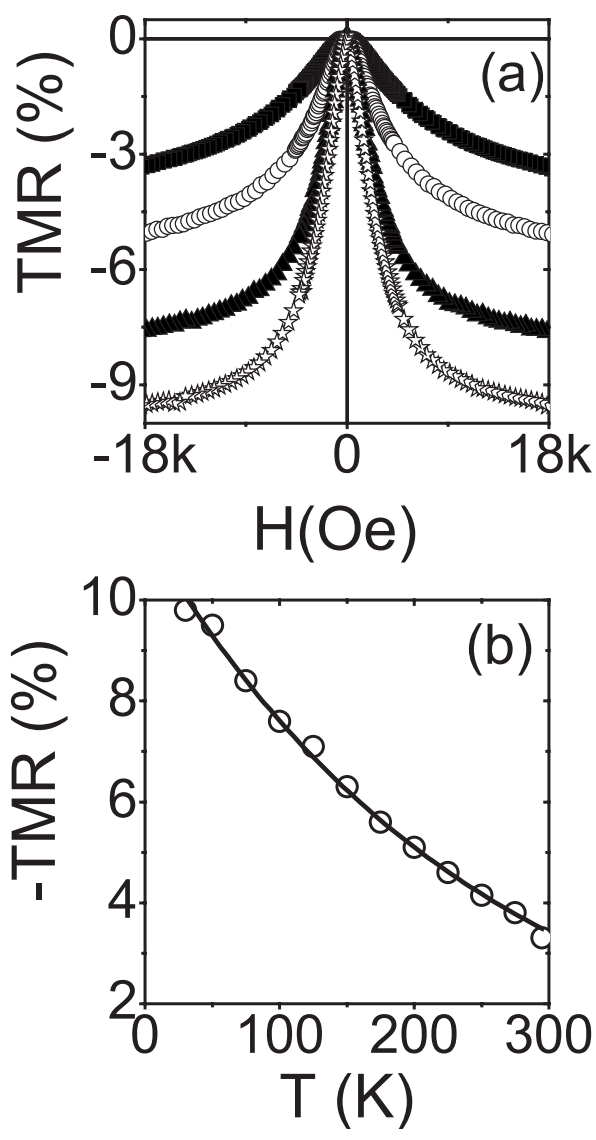

FIG. 3. (a) TMR in $T$-geometry for DMIM with $\mathrm{t}=0.61 \mathrm{~nm}$ at $T=295 \mathrm{~K}$ (full squares), $T=200 \mathrm{~K}$ (open circles), $T=100 \mathrm{~K}$ (full triangles), and $T$ $=50 \mathrm{~K}$ (open stars), (b) Temperature dependence of TMR at $\mathrm{H}=18 \mathrm{kOe}$ (open circles). Solid line is the best fit using equation TMR $=-P^{2} m^{2} \exp \left(-k_{B} T / E_{\mathrm{mr}}\right)$ with parameters $P=0.336$ and $E_{\mathrm{mr}} / k_{B}=474 \mathrm{~K}$.

expression is written in the form $\mathrm{TMR}=-P^{2} m^{2}(1$ $+\sqrt{B / T}) \exp \left(-k_{B} T / E_{\mathrm{mr}}\right)$, and can be reduced to TMR $\approx-P^{2} m^{2} \exp \left(-k_{B} T / E_{\mathrm{mr}}\right)$ outside the Coulomb blockade regime, where $k_{B} T>E_{\mathrm{c}}$. The experimental dependence of TMR versus temperature for the sample with $\mathrm{t}=0.61 \mathrm{~nm}$ was fitted using this formula (Fig. 3). The values for $m^{2}$ were taken from magnetization results reported earlier for the same sample. ${ }^{16}$ The best fit was achieved using $P$ $=0.336 \pm 0.002$ and $E_{\mathrm{mr}} / k_{B}=474 \pm 19 \mathrm{~K}$. These values are similar to those derived for $\mathrm{Fe}-\mathrm{Al}_{2} \mathrm{O}_{3}$ granular cermets $(P$ $=0.375$ and $\left.E_{\mathrm{mr}} / k_{B}=270 \mathrm{~K}\right){ }^{23}$ The dramatic increase of TMR at low $T$ characteristic of insulating granular systems $^{22-24}$ has not been observed in our samples due to their high resistance below $30 \mathrm{~K}(\mathrm{R}>3 \mathrm{G} \Omega)$.

The values of spin polarization for ferromagnetic nanogranules structures derived in our work and those published earlier $^{22-24}$ are low compared with the corresponding bulk metals $(P=0.44$ in the case of $\mathrm{Fe}$, Ref. 25). This reduction can be explained taking into account formation of a thin $\mathrm{FeO}_{\mathrm{x}}$ layer on the surface of the grains. For Fe-based granular systems the presence of $\mathrm{FeO}_{\mathrm{x}}$ layer causes the reduction of magnetic moment per $\mathrm{Fe}$ atom and leads to increase in the coercive field at low temperature. ${ }^{26}$ Similar effect was observed in our films. ${ }^{16,27}$ Extended x-ray absorption fine structure and Mössbauer measurements give evidence of $\mathrm{Fe}^{2+}$ and $\mathrm{Fe}^{3+}$ ions located near the interface $\mathrm{Fe}-\mathrm{FeO}$ in $\mathrm{Fe} / \mathrm{ZrO}_{2}$
DMIMs. ${ }^{28}$ Most probably, these $\mathrm{Fe}$ ions contribute to the depolarization of the tunneling electrons due to spin-flip scattering events. ${ }^{28}$

In summary, we have shown that electrical transport in $\mathrm{Fe}(\mathrm{t}) / \mathrm{MgO}(3 \mathrm{~nm})$ multilayers depends critically on the structure of the Fe films, being governed by metallic conduction for continuous $\mathrm{Fe}$ layers $(\mathrm{t} \geq 0.81 \mathrm{~nm})$ and by spindependent tunneling between $\mathrm{Fe}$ grains in the case of discontinuous layers $(\mathrm{t}<0.81 \mathrm{~nm})$. The obtained TMR ratios at room temperature are the highest reported up to date in $\mathrm{Fe} /$ $\mathrm{MgO}$ DMIMs. Modifications of technological process and improvement of the DMIM structure can increase the TMR values, as preliminary results obtained by using $\mathrm{MgO}(001)$ substrates confirm (not shown here). The thermal dependence of TMR was satisfactorily explained using a modified Mitani's model, based on the occurrence of spin-flip scattering processes affecting the electrons involved in spindependent tunneling, considering a decrease of the spin polarization due to the presence of $\mathrm{FeO}_{\mathrm{x}}$ shell surrounding $\mathrm{Fe}$ particles.

\section{ACKNOWLEDGMENTS}

Financial support by Spanish Ministry of Science (through Project Nos. MAT2008-06567-C02 and CIT420000-2008-19 including FEDER funding) and by the Aragón Regional Government (through Project Nos. E26 and PI059/08) is acknowledged.

${ }^{1}$ S. A. Wolf, D. D. Awschalom, R. A. Buhrman, J. M. Daughton, S. von Molnar, M. L. Roukes, A. Y. Chtchelkanova, and D. M. Treger, Science 294, 1488 (2001).

${ }^{2}$ S. Kirkpatrick, Rev. Mod. Phys. 45, 574 (1973).

${ }^{3}$ B. Abeles, P. Sheng, M. D. Coutts, and Y. Arie, Adv. Phys. 24, 407 (1975). ${ }^{4}$ H. Fujimori, S. Ohnuma, N. Kobayashi, and T. Masumoto, J. Magn. Magn. Mater. 304, 32 (2006).

${ }^{5}$ A. Vovk, J. Q. Wang, J. He, W. Zhou, A. Pogoriliy, O. Shypil', A. Kravets, and H. Khan, J. Appl. Phys. 91, 10017 (2002).

${ }^{6}$ G. N. Kakazei, Yu. G. Pogorelov, A. M. L. Lopes, J. B. Sousa, S. Cardoso, P. P. Freitas, M. M. Pereira de Azevedo, and E. Snoeck, J. Appl. Phys. 90, 4044 (2001)

${ }^{7}$ S. Yuasa, T. Nagahama, A. Fukushima, Y. Suzuki, and K. Ando, Nature Mater. 3, 868 (2004)

${ }^{8}$ Y. M. Lee, J. Hayakasawa, S. Ikeda, F. Matsukura, and H. Ohno, Appl. Phys. Lett. 90, 212507 (2007).

${ }^{9}$ X. Xu, V. M. Karpan, K. Xia, M. Zwierzycki, I. Marushchenco, and P. J. Kelly, Phys. Rev. B 73, 180402(R) (2006).

${ }^{10}$ P. Bose, A. Ernst, I. Mertig, and J. Henk, Phys. Rev. B 78, 092403 (2008).

${ }^{11}$ S. Maekawa and U. Gäfvert, IEEE Trans. Magn. 18, 707 (1982).

${ }^{12}$ J. Inoue and S. Maekawa, Phys. Rev. B 53, R11927 (1996).

${ }^{13}$ J. S. Slonczewski, Phys. Rev. B 39, 6995 (1989).

${ }^{14}$ J. Mathon and A. Umerski, Phys. Rev. B 63, 220403 (2001).

${ }^{15}$ M. Arita, K. Wakasugi, K. Ohta, K. Hamada, T. Takahashi, and J. B. Choi, Microelectron. Eng. 85, 2445 (2008).

${ }^{16}$ A. García-García, A. Vovk, J. A. Pardo, P. Štrichovanec, C. Magén, E. Snoeck, P. A. Algarabel, J. M. De Teresa, L. Morellón, and M. R. Ibarra, J. Appl. Phys. 105, 063909 (2009).

${ }^{17}$ C. Liu, Y. Park, and S. D. Bader, J. Magn. Magn. Mater. 111, L225 (1992).

${ }^{18}$ S. Sangiao, L. Morellón, G. Simón, J. M. De Teresa, J. A. Pardo, J. Arbiol, and M. R. Ibarra, Phys. Rev. B 79, 014431 (2009).

${ }^{19}$ S. Honda, T. Okada, M. Nawate, and M. Tokumoto, Phys. Rev. B 56, 14566 (1997).

${ }^{20}$ A. Ya. Vovk, V. O. Golub, L. Malkinski, A. F. Kravets, A. M. Pogorily, and O. V. Shypil, J. Magn. Magn. Mater. 272-276, E1403 (2004).

${ }^{21}$ T. R. McGuire and R. I. Potter, IEEE Trans. Magn. 11, 1018 (1975).

${ }^{22}$ S. Mitani, S. Takahashi, K. Takanashi, K. Yakushiji, S. Maekawa, and H. 
Fujimori, Phys. Rev. Lett. 81, 2799 (1998).

${ }^{23}$ T. Zhu and Y. J. Wang, Phys. Rev. B 60, 11918 (1999).

${ }^{24}$ B. J. Hattink, M. García del Muro, Z. Konstantinović, X. Batlle, A. Labarta, and M. Varela, Phys. Rev. B 73, 045418 (2006).

${ }^{25}$ P. M. Tedrow and R. Meservey, Phys. Rev. B 7, 318 (1973); R. Meservey and P. M. Tedrow, Phys. Rep. 238, 173 (1994).

${ }^{26}$ N. M. Dempsey, L. Ranno, D. Givord, J. Gonzalo, R. Serna, G. T. Fei, A.
K. Petford-Long, R. C. Doole, and D. E. Hole, J. Appl. Phys. 90, 6268 (2001).

${ }^{27}$ A. García-García, A. Vovk, P. Štrichovanec, J. A. Pardo, C. Magén, P. A. Algarabel, J. M. De Teresa, L. Morellón, and M. R. Ibarra, J. Phys.: Condens. Matter 22, 056003 (2010).

${ }^{28}$ P. Auric, J. S. Micha, O. Proux, L. Giacomoni, and J. R. Regnard, J. Magn. Magn. Mater. 217, 175 (2000). 